Acta Astronautica, Volume 128, November-December 2016, Pages 343-349

FAILURE ANALYSIS OF SATELLITE SUBSYSTEMS TO DEFINE SUITABLE DE-ORBIT DEVICES

\author{
Ms. Chiara Palla \\ Cranfield University, United Kingdom, c.palla@cranfield.ac.uk \\ Mr. Moreno Peroni \\ Italian Air Force, Flight Test Wing, Aero-Space System Engineering Group, Italy, \\ moreno.peroni@aeronautica.difesa.it \\ Dr. Jennifer Kingston \\ Cranfield University, United Kingdom, j.kingston@cranfield.ac.uk
}

Space missions in Low Earth Orbit (LEO) are severely affected by the build-up of orbital debris. A key practice, to be compliant with IADC (Inter-Agency Space Debris Coordination Committee) mitigation guidelines, is the removal of space systems that interfere with the LEO region not later than 25 years after the End of Mission. It is important to note that the current guidelines are not generally legally binding, even if different Space Agencies are now looking at the compliance for their missions. If the guidelines will change in law, it will be mandatory to have a post mission disposal strategy for all satellites, including micro and smaller classes.

A potential increased number of these satellites is confirmed by different projections, in particular in the commercial sector. Micro and smaller spacecraft are, in general, not provided with propulsion capabilities to achieve a controlled re-entry, so they need different de-orbit disposal methods.

When considering the utility of different debris mitigation methods, it is useful to understand which spacecraft subsystems are most likely to fail and how this may affect the operation of a de-orbit system. This also helps the consideration of which components are the most relevant or should be redundant depending on the satellite mass class.

This work is based on a sample of LEO and MEO satellites launched between January 2000 and December 2014 with mass lower than $1000 \mathrm{~kg}$. Failure analysis of satellite subsystems is performed by means of the Kaplan-Meier survival analysis; the parametric fits are conducted with Weibull distributions. The study is carried out by using the satellite database SpaceTrak ${ }^{\mathrm{TM}}$ which provides anomalies, failures, and trends information for spacecraft subsystems and launch vehicles. The database identifies five states for each satellite subsystem: three degraded states, one fully operational state, and one failed state (complete failure).

The results obtained can guide the identification of the activation procedure for a de-orbit strategy and the level of integration it should have with the host satellite in order to be activated before a total failure.

At Cranfield Space Research Centre two different solutions have already been developed as de-orbit sail payloads for micro satellites (Icarus-1 on TechDemoSat-1 and Icarus-3 on Carbonite-1 currently on-orbit, DOM for future ESA ESEO mission). This study will provide a useful input to improve and refine the current de-orbit concepts for future satellite missions.

\section{INTRODUCTION}

Orbital debris in the LEO region continues to increase, posing a major risk to future space missions and to the exploitation of key orbits. The IADC (InterAgency Space Debris Coordination Committee) has established a set of guidelines [1] to ensure the future safe and sustainable use of this region; among them, the post-mission disposal within 25 years, for space systems in LEO, has been agreed as a key practice.

A general intention to change the Space Debris Mitigation guidelines in law in the near future can be perceived within the main space agencies and in different countries: ESA for example has recently adopted the ISO 24113 [2] as requirements for its missions [3], as well as in France the French Space Operations Act has been mandatory since 2010 [4].

To make provision for these anticipated requirements, it will be mandatory to have post mission disposal strategies for all satellites, including micro and smaller classes. Micro and smaller spacecraft are, in general, not provided with propulsion capabilities to achieve a controlled re-entry, so they need different deorbit disposal methods.

When evaluating the utility of different debris mitigation methods, it is useful to understand which spacecraft subsystems are most likely to fail and how this may affect the operation of a de-orbit system. This also helps the consideration of which components are the most relevant or should be redundant depending on the satellite mass class. 


\section{I.I De-orbit strategies}

After the End of Mission the spacecraft disposal can be achieved with different de-orbit methods. The deorbit strategies for LEO clearance within 25 years can be divided in two main categories: active and passive [5].

It is worth noting also the possibility of using the natural decay, when End of Mission (EoM) disposal manoeuvre is not required.

Active de-orbit strategies make use of propulsion to accomplish the disposal. They need the spacecraft bus subsystems to be fully operational at the EoM, from the power supply to the attitude control on-board and TT\&C. Among the active methods there are chemical propulsion and electric propulsion. On-board propulsion is a space-proven technology; however this strategy can strongly limit operational lifetime, as fuel mass is dedicated to the de-orbiting. In addition the propulsion subsystem can experience significant degradation on orbit and if it (partially) fails, it is likely to have major effect on mission capability [6].

D-Orbit ${ }^{\mathrm{TM}}$ smart propulsive device [7] can also be included in the propulsive methods; however this decommissioning device, currently at qualification level, is quite independent from the spacecraft itself, in fact it has its own electronics, communications, attitude and propulsion subsystems [8]. This device just needs a power line from the spacecraft host to charge one of the two batteries provided. The de-orbit manoeuvre is accomplished by means of a solid rocket motor [9].

Passive disposal strategies make use of devices to enhance the exploitation of external forces and they are independent of spacecraft propulsion capabilities. These methods take advantage of electromagnetic drag (tethers) or aerodynamic drag (drag augmentation devices) as the de-orbit force.

The drag augmentation concepts considered in this work are: Boom-supported film aerobrake and Inflation ultrathin envelope (i.e. balloon) [10]. Cranfield University has recent experience in design and development of aerodynamic drag-based de-orbit devices of the aerobrake concept type (Icarus-1 [11], DOM [12], Icarus-3 [13]).

\section{METHODOLOGY}

This work is based on a sample of recently launched satellites belonging to small mass classes. Failure analysis of satellite subsystems is performed using a statistical approach. The study is carried out by using the satellite database SpaceTrak ${ }^{\mathrm{TM}}$ which provides anomalies and failures for spacecraft subsystems.

The process followed in this work has been firstly to extract the failure data per spacecraft, developing a separate database; then to perform a Kaplan-Meier analysis followed by Weibull distribution estimation in MATLAB for the different subsystems reliabilities; finally to combine the different subsystem reliabilities depending on the de-orbit strategies (presented in I.I). Following the reliabilities study a trade-off analysis to guide in the de-orbit methods selection has been performed.

\section{II.I Spacecraft Failures Database}

The sample extracted from SpaceTrak ${ }^{\mathrm{TM}}$ is composed of satellites in LEO, MEO, and Elliptical orbits, with mass lower than $1000 \mathrm{~kg}$, launched between the $1^{\text {st }}$ January 2000 and the $31^{\text {st }}$ December 2014. MEO and Elliptical orbits satellites have been added to the sample to obtain more failures data considering that their design and level of technology is similar to the LEO ones.

Spacecraft above $1000 \mathrm{~kg}$ have not been taken into account as they are considered to require targeted reentry due to the likelihood that some components may survive the re-entry. Suborbital vehicles and cargo missions have also not been included since they are out of the scope of this work.

For each spacecraft $(\mathrm{S} / \mathrm{C})$ in the sample the key parameters collected from the database are: (1) S/C name, (2) S/C bus, (3) Launch Date, (4) Mass, (5) Failure Event Type and Date, (6) Retirement Date, (7) Re-Entry Date. The last three parameters obviously don't necessarily occur for all the spacecraft.

In addition a new parameter, not available in the database, has been added: this confirms if a propulsion subsystem is on-board or not. Some assumptions have been taken to speed up the research: $\mathrm{S} / \mathrm{C}$ with mass lower than $3 \mathrm{~kg}$ have been considered with no propulsion. This is based on analysis of available information on proposed $\mathrm{S} / \mathrm{C}<3 \mathrm{~kg}$ : the majority do not have chemical propulsion sufficient to perform de-orbit; moreover most cubesats do not carry a significant propulsion subsystem. S/C with mass higher than 500 $\mathrm{kg}$ instead have been considered equipped with propulsion subsystem.

The completion of the database with information about the propulsion subsystem has been performed using different sources, in particular Gunter's Space Page [14], Earth Observation Portal [15], SatFlare [16], RussianSpaceWeb [17].

\begin{tabular}{|c|c|c|}
\hline Spacecraft & Propulsion & No propulsion \\
\hline 798 & 380 & 418 \\
\hline
\end{tabular}
propulsion subsystem or not.

The SpaceTrak ${ }^{\mathrm{TM}}$ database identifies five states for each satellite subsystem [18]: one fully operational state, three degraded states (Class IV, Class III-LR, 
Class II), and one complete failure state (Class I) that brings the satellite to retirement. For the purpose of this study Class I (i.e. "death" of the satellite) and Class II (i.e. major failure on the satellite subsystem) have been considered.

As stated in the SpaceTrak ${ }^{\mathrm{TM}}$ User Manual [18] the failures and anomalies in the database are only included during design life, up to a maximum of 15 years, whichever is the shorter period.

Starting from the subsystem failure classification of SpaceTrak ${ }^{\mathrm{TM}}$ five different failure typology groups have been derived:

- TTC: telemetry, tracking, and command together with control processor failures and OBDH issues;

- POW: electrical distribution, batteries, and solar arrays issues;

- ATT: gyro, reaction wheel, attitude control, thrusters, and fuel anomalies;

- $\mathrm{MECH}$ : mechanisms, structures, thermal, and antenna deployment failures;

- PAY \& UNK: payload and unknown problems

The satellites have been divided into classes depending on their mass (nano, micro, mini, small); however due to the small number of failures new broader classes have been used:

- Up to $1 \mathrm{~kg}$ : equivalent to picosatellites and lighter;

- 1-10 kg: corresponding to nanosatellites;

- 10-100 kg: corresponding exactly to the microsatellites as classified by SpaceTrak ${ }^{\mathrm{TM}}$;

- 100-1000 kg: equivalent to mini and small satellites, to be noted that for satellites without propulsion the upper limit is $500 \mathrm{~kg}$.

Despite this new division it was found impossible to perform the statistical analysis for some typology groups and for some mass classes because there were not enough failure data.

II.II Kaplan-Meier Analysis and Weibull Distribution

Kaplan-Meier analysis, developed in 1958 [19], is largely used in medicine to estimate a survival curve from a population sample; a good example is given by Tripepi [20].

One of the key features of this analysis is the management of incomplete observations, i.e. the right- censored data. This happens when no information is available about one element of the sample after a specific time, for example a patient who quits the treatment. The analysis also works when subjects begin the study in different time instants, because their lifetimes can be shifted to a common zero position and then compared.

These relevant aspects fit perfectly with the sample of satellite subsystems to analyse. In fact the dataset of the satellites is censored, this is when the satellite is retired or re-entered or at the end of the observation period; moreover the subjects - the satellites - are launched and then on-orbit in different times.

As can be seen from the example in Table 2 the probability $\mathrm{R}(\mathrm{t})$ of surviving at any time $\mathrm{t}$ (or the Reliability at time $\mathrm{t}$ ) is calculated from the cumulative probability $\left(1-\mathrm{F}\left(\mathrm{t}_{\mathrm{i}}\right)\right)$ of surviving to each of the previous time intervals, i.e. the product of current and previous probabilities. The failure probability $\mathrm{F}(\mathrm{t})$ is then given by $1-\mathrm{R}(\mathrm{t})$.

The data of cumulative reliability presented in the previous table are summarized in Fig. 1 which makes use of the Kaplan-Meier plot.

Fig. 1: Example of Kaplan-Meier plot with MATLAB.

The Kaplan-Meier estimator is a non-parametric analysis. So from the step function it is difficult to calculate the failure rate. A link with a parametric function is then necessary. For the purpose of this analysis the curve that fits the reliability data is the Weibull distribution. To achieve this, the MATLAB software has been used; once a table like Table 2 is given it can produce both the Kaplan-Meier plot and estimate the Weibull parameters for that particular step function.

The Weibull distribution is widely used in science and engineering to describe components' lives (or failures), strengths of brittle materials, etc. It is an example of a minimum extreme value distribution.

Following the previous work performed by Saleh and Castet [21] once the empirical data of subsystems failures are collected, then the Weibull distribution is used for the data fit and to extrapolate future trend (see

\begin{tabular}{cccccccc}
\hline Time & Lost & Censored & Subjects & $\mathrm{F}\left(\mathrm{t}_{\mathrm{i}}\right)$ & $1-\mathrm{F}\left(\mathrm{t}_{\mathrm{i}}\right)$ & $\mathrm{R}(\mathrm{t})$ & $\mathrm{F}(\mathrm{t})$ \\
\hline 2 & 1 & 0 & 10 & 0.100 & 0.900 & 0.900 & 0.100 \\
6 & 1 & 0 & 9 & 0.111 & 0.888 & 0.800 & 0.200 \\
7 & 1 & 1 & 7 & 0.143 & 0.857 & 0.686 & 0.314 \\
8 & 1 & 0 & 6 & 0.167 & 0.833 & 0.571 & 0.429 \\
9 & 2 & 0 & 5 & 0.400 & 0.600 & 0.343 & 0.657 \\
\hline
\end{tabular}

Table 2: Kaplan-Meier table example: $\mathrm{F}(\mathrm{t})$ is the Failure probability, $\mathrm{R}(\mathrm{t})$ is the Reliability. Note $\mathrm{t}_{\mathrm{i}}$ is the time interval. 
Fig. 2).

Saleh and Castet considered a database of 1584 Earth-orbiting satellites launched between January 1990 and October 2008, including also GEO satellites [22]. Their work shows that a Weibull distribution fits the failure behaviour of the S/C subsystems well; however the sample is considered too broad for the purposes described in this paper, as the application, the development, testing and also the operational environment of micro and smaller satellites is significantly different compared to the geostationary satellites (including meteorological spacecraft in GEO).

In this case instead the focus is on LEO satellites and the reliability analysis is performed on the subsystems divided by mass class and S/C equipped with propulsion or not, because the purpose is to assist in the selection of the de-orbit method (and this can vary depending on the size and design of the satellite).

Fig. 2: Example of Kaplan-Meier plot and corresponding Weibull distribution for Attitude subsystem (ATT) of S/C with propulsion, mass range $100-1000 \mathrm{~kg}$.

The following equation represents the Weibull reliability function for the satellite or subsystem reliability. $\beta$ is the shape parameter (dimensionless) and $\theta$ is the scale parameter (days).

$$
R(t)=e^{-\left(\frac{t}{\theta}\right)^{\beta}}
$$

The $\beta$ parameter gives insight of the failure trend: if $\beta<1$ the rate decrease with time (this is the case of infant mortality), if $\beta>1$ the failures will increase with time.

The $\theta$ parameter gives an estimation of the time in which the component has a $63.2 \%$ probability to fail [23]. Another important aspect of the Weibull function is that even if the sample is relatively small it can give good confidence when used for extrapolation [21].

\section{II.III Subsystems vs. De-orbit Strategies}

The last phase of the study combines the subsystems (typology groups) reliabilities and matches them with the de-orbit strategies.

The de-orbit strategies considered are propulsive deorbit (chemical or electrical), drag augmentation devices, electromagnetic tether, and solid propulsion but independent from the spacecraft (D-orbit ${ }^{\mathrm{TM}}$ propulsive device [7]).

The selection and combination of subsystems reliabilities is related to the following question: "Which subsystems are required to be functional at EoM for the disposal strategy selected?"
After a technical evaluation the combinations of subsystems presented in Fig. 3 have been analysed.

Fig. 3: Combinations of subsystems vs. de-orbit strategies.

The subsystems taken into account belong only to the satellite host and not to the de-orbit methods; for example, a passive drag device will have specific mechanisms to deploy the sail, but the $\mathrm{MECH}$ subsystem of the spacecraft is not needed to activate the deployment and so it is not included in the reliability combination.

All the combinations considered are series systems; this means that the overall system reliability is the product of the individual subsystem reliabilities, assuming that the individual reliabilities are independent from each other [23].

$$
R_{\text {syst }}=R_{1} \times R_{2} \times \ldots \times R_{n}=\prod_{i=1}^{n} R_{i}
$$

\section{RESULTS}

Before going into the detailed subsystems reliabilities the spacecraft overall reliabilities have been calculated (these consider also failures unknown or related to the payload).

Fig. 4: Weibull reliabilities comparison for S/C by mass classes, the different time scale to simulate the Weibull distributions is derived from a sample of re-entered spacecraft (with or without propulsion subsystem).

In Fig. 4 it is interesting to see the stronger infant mortality for smaller spacecraft without propulsion, as expected.

As can be seen, spacecraft without propulsion have a trend that stops at 2.5 years, while the bigger ones with propulsion it stops at 10 years. The time scale selected for the Weibull distributions have been in fact calculated from a sample of re-entered spacecraft (177 in total) in the period 2000-2014 considering the third quartile and the standard deviation of their lifetimes (separating data into S/C with propulsion 67 and S/C without it 110).

The different timespans allow taking into account the design life of the missions and so the reliability performances. The same time scale is then used for the subsystems reliabilities. 


\begin{tabular}{cccc}
\hline Subsystem & Mass classes [kg] & $\theta$ [days] & $\beta[-]$ \\
\hline Spacecraft & Propulsion 100-1000 & $9.3126 \times 10^{4}$ & 0.6733 \\
& No propulsion 1-10 & $2.7441 \times 10^{7}$ & 0.3456 \\
& No propulsion 10-100 & $2.1781 \times 10^{6}$ & 0.4077 \\
ATT & Propulsion 100-1000 & $1.5427 \times 10^{6}$ & 0.3480 \\
& No propulsion 1-10 & $/$ & $/$ \\
& No propulsion 10-100 & $/$ & $/$ \\
MECH & Propulsion 100-1000 & $2.0766 \times 10^{10}$ & 0.2072 \\
& No propulsion 1-10 & $7.6463 \times 10^{7}$ & 0.2999 \\
& No propulsion 10-100 & $/$ & $/$ \\
TTC & Propulsion 100-1000 & $5.4928 \times 10^{5}$ & 0.5353 \\
& No propulsion 1-10 & $1.1193 \times 10^{10}$ & 0.2458 \\
& No propulsion 10-100 & $7.9801 \times 10^{5}$ & 0.5017 \\
POW & Propulsion 100-1000 & $6.3973 \times 10^{4}$ & 0.9846 \\
& No propulsion 1-10 & $1.2799 \times 10^{6}$ & 0.4112 \\
& No propulsion 10-100 & $8.8481 \times 10^{5}$ & 0.4548 \\
\hline
\end{tabular}

Table 3: Weibull fit parameters extrapolated by MATLAB.

III.I Subsystems Reliabilities

In this section the Weibull reliabilities for the different subsystems and mass classes obtained by means of the statistical analysis are presented.

It is relevant to note that the reliabilities calculated do not take into account human factors and design errors but simply the anomaly or part/component failure which cause the spacecraft subsystem (Class II) or mission (Class I) to fail. The failures can be due to moving parts, solid-state electronics, or other components, in a number of different subsystems; in this way the inherent reliability is related to the technology type. However, it must be noted that it is the end result that is critical, for instance if the AOCS fails it is not important if it failed due to a mechanism or other, it still cannot be used for the de-orbit.

As can be seen some mass classes are missing (e.g.: $\mathrm{S} / \mathrm{C}$ without propulsion with mass $>100 \mathrm{~kg}$ ), this is due to the lack of relevant data for that class.

The attitude subsystem for spacecraft with propulsion and mass class $100-1000 \mathrm{~kg}$ is the only one obtained for the different groups analysed. Although a comparison is not possible, it is interesting to look at the reliability decrease, which is below $90 \%$ just after 6 years of lifetime. This is relevant to consider when the disposal reliability threshold is calculated as it requires to maintain a probability of 0.9 for a successful disposal [24]. For this reason if the de-orbit strategy selected needs the attitude subsystem to work at the time of disposal it is better to have a reliability far above the $90 \%$ as this is going to be combined with other probabilities (e.g.: reliability of the de-orbit strategy itself, probability of achieving the disposal, etc.).

Fig. 5: Weibull reliability for Attitude subsystem (ATT) of $\mathrm{S} / \mathrm{C}$ with propulsion.

Fig. 6: Weibull reliabilities comparison for Mechanisms subsystem $(\mathrm{MECH})$ by mass classes.

In the case of mechanism and thermal subsystem the trends are always above $96 \%$ for the time scale considered.

TTC and POW subsystems (see Fig. 7, 8) seem to have higher reliability compared to the ATT subsystem for spacecraft with propulsion. Spacecraft not equipped with propulsion have reliability above $95 \%$ for the time scale considered, with TTC more reliable than the POW. From the POW trends it is clear that bigger spacecraft with more redundancy and higher budget availability have more reliable Power subsystems, however the failure rate has a constant decreasing rate.

Both TTC and POW subsystems are crucial for the accomplishment of a space mission, and they are for the EoM disposal as well, because the capability of achieving the disposal by the de-orbit strategy is strongly dependent on them. 
Fig. 7: Weibull reliabilities comparison for TT\&C subsystem (TTC) by mass classes.

Fig. 8: Weibull reliabilities comparison for Power subsystem (POW) by mass classes.

\section{III.II Combined Reliabilities}

As mentioned in the methodology section the different combinations are subsystems in series. The combinations evaluated and the de-orbit strategies considered are the ones presented in Fig. 3.

In particular it is interesting to look at the difference in the trends when adding in series the ATT subsystem with respect to the case of TTC \& POW combination only (see Fig. 9). This suggests to select a different deorbit strategy than the propulsive de-orbit, in particular in the case of long missions. An option could be the drag-augmentation devices, which need only power supply and TT\&C to operate.

The passive drag device seems definitely a good solution for S/C without propulsion, if just the reliability trends are considered (see Fig. 9). The combined reliability of the subsystems (TTC \& POW) required for this strategy is always above $92 \%$ for the selected time scale and in general above $90 \%$ up to 7 years.

The trends presented in Fig. 10 are useful when evaluating the D-Orbit ${ }^{\mathrm{TM}}$ propulsive device, as mentioned before this strategy needs only the power line from the spacecraft host to charge its own batteries.

Clearly shorter mission duration is likely to lead to a higher reliability for a given set of spacecraft equipment, due to the decreasing reliability over time. An aim of this work is to inform decisions about suitable choice of de-orbit approach, and also appropriate timing of the end of a mission.

Fig. 9: Weibull combined reliabilities TTC \& POW \& ATT vs. TTC \& POW by mass classes. These trends are useful for the drag-augmentation device.

Fig. 10: Weibull combined reliabilities TTC \& POW \& ATT vs. POW only by mass classes. These trends are useful for the D-Orbit ${ }^{\mathrm{TM}}$ propulsive device.

\section{III.III Trade-off Analysis}

Deciding which type of de-orbit strategy can fit with a specific LEO mission is a complicated matter, with many variables to take into account. For this reason a trade-off analysis has been performed.

The reliability of the subsystems is indeed not the only factor to consider for the de-orbit strategy selection. Aspects related to the technology of the disposal strategy (e.g.: TRL, physical properties, reliability of the disposal method itself), the mission (e.g.: orbit parameters, duration), and the spacecraft need to be considered.

The aim of the paper is to assess the impact of subsystem reliabilities on the function of different deorbit methods. Clearly the reliability and safety of the de-orbit methods themselves are also of great importance in achieving an effective de-orbit solution, however the assessment of these reliabilities is outside the scope of this work.

In this analysis the focus is on the application and integration of the de-orbit strategy with respect to the spacecraft host.

The orbit altitude is not considered, the assumption is an altitude where all the de-orbit strategies can be used. However, it must be clear that some de-orbit technologies are only effective below certain orbit altitudes (e.g.: drag augmentation concepts) or at specific inclination (e.g.: electric tether).

For each disposal strategy (see paragraph II.III) the trade-off parameters are:

- Feasibility: about the implementation and integration of the de-orbit strategy with the host spacecraft ( 5 = very easily);

- Mass: incidence of the de-orbit strategy mass to the spacecraft mass ( $5=$ no incidence);

- TRL: in space missions and tests $(5=\operatorname{good}$ heritage equivalent to TRL 8/9).

In addition the combined reliabilities relevant for each strategy at three time periods $(2.5,5$, and 10 years $)$ have been included. These have been discussed in the Results section.

The minimum value, which is 0 , has been assigned to the lowest combined reliability detected (77\% for the TTC \& POW \& ATT for S/C with propulsion in the $100-1000 \mathrm{~kg}$ mass class at 10 years); the maximum value, which is 5 , to the highest reliability observed (98\% POW for S/C with propulsion in $100-1000 \mathrm{~kg}$ mass class at 2.5 years).

The trade-off is aimed at LEO spacecraft but uses the overall reliability data derived previously, which includes MEO spacecraft as discussed before in II.I section.

Considering the main purpose of this analysis, the parameters are selected to be the most relevant for evaluating suitability for integration with the spacecraft host. 


\begin{tabular}{c|lccccccccc}
\hline Class & $\begin{array}{l}\text { De-orbit } \\
\text { strategy }\end{array}$ & $\begin{array}{c}\text { Feasi } \\
\text { bility }\end{array}$ & Mass & TRL & $\begin{array}{c}\text { R(t) at } \\
2.5 \text { ys }\end{array}$ & TOT & $\begin{array}{c}\text { R(t) at } \\
5 \text { ys }\end{array}$ & TOT & $\begin{array}{c}\text { R(t) at } \\
10 \text { ys }\end{array}$ & TOT \\
\hline Prop & Propulsive & 5 & 4 & 5 & 2.6 & $\mathbf{1 6 . 6}$ & 1.4 & $\mathbf{1 5 . 4}$ & 0 & $\mathbf{1 4}$ \\
$\mathbf{1 0 0 - 1 0 0 0}$ & Electr. tether & 2 & 3 & 2.5 & 4.3 & $\mathbf{1 1 . 8}$ & 3.6 & $\mathbf{1 1 . 1}$ & 2.4 & $\mathbf{9 . 9}$ \\
kg & Film aerobrake & 4 & 3 & 4 & 4.3 & $\mathbf{1 5 . 3}$ & 3.6 & $\mathbf{1 4 . 6}$ & 2.4 & $\mathbf{1 3 . 4}$ \\
& Inflatable env. & 2 & 4 & 1.5 & 4.3 & $\mathbf{1 1 . 8}$ & 3.6 & $\mathbf{1 1 . 1}$ & 2.4 & $\mathbf{9 . 9}$ \\
& Prop. D-Orbit & 5 & 2 & 3 & 5 & $\mathbf{1 5}$ & 4.8 & $\mathbf{1 4 . 8}$ & 4 & $\mathbf{1 4}$ \\
No prop & Electr. tether & 2.5 & 3 & 2.5 & 3.8 & $\mathbf{1 1 . 8}$ & & & & \\
$\mathbf{1 - 1 0}$ kg & Film aerobrake & 4 & 3 & 4 & 3.8 & $\mathbf{1 4 . 8}$ & & & & \\
& Inflatable env. & 1 & 2 & 1.5 & 3.8 & $\mathbf{8 . 3}$ & & & & \\
No prop & Electr. tether & 3.5 & 4 & 2.5 & 3.6 & $\mathbf{1 3 . 6}$ & & & & \\
$\mathbf{1 0 - 1 0 0}$ kg & Film aerobrake & 4 & 4 & 4 & 3.6 & $\mathbf{1 5 . 6}$ & & & & \\
& Inflatable env. & 3 & 3 & 1.5 & 3.6 & $\mathbf{1 1 . 1}$ & & & & \\
& Prop. D-Orbit & 4 & 3 & 3 & 4.5 & $\mathbf{1 4 . 5}$ & & & & \\
\hline
\end{tabular}

Table 4: Trade-off table for decision making in the selection of the de-orbit strategy by S/C classes.

For bigger spacecraft with propulsion the conventional de-orbit propulsive method seems still the best solution; however the trade-off results show a drop over time for this strategy. In fact despite the high ranking of the parameters not related with the reliability, a crucial result of the study is that the attitude subsystem reliability decreases the final result over time. As can be seen it is then worthy consider the D-Orbit ${ }^{\mathrm{TM}}$ propulsive device as a good alternative, in particular for long missions, provided an appropriate reliability of such a device can be demonstrated. Indeed, the TRL parameter for this disposal device has been set lower than other methods because of the current status of the technology.

The drag-augmentation device, in particular the boom supported film aerobrake, results the best solution for nano and microsatellites without propulsion, with good values as well for spacecraft with propulsion.

\section{CONCLUSIONS}

The results obtained can guide the identification of the activation procedure for a de-orbit strategy and the level of integration it should have with the host satellite in order to be activated before a total failure or before the failure of the subsystems necessary for its operation.

The reliability analyses accomplished give a more detailed insight into the LEO satellites compared to the work performed by Saleh and Castet. The purpose is in fact to guide the selection of the de-orbit strategy.

Spacecraft equipped with a propulsion subsystem show a higher reliability than the ones without; however the attitude subsystem has the worst reliability and this is particularly relevant for the selection of the disposal method. On the other hand the power subsystem seems very reliable for the class $100-1000 \mathrm{~kg}$ with propulsion, but for smaller satellites without propulsion instead it is the worst.

The reliabilities calculated take into account the anomaly or part/component failure which causes the subsystem to fail, on the other hand human factors and system engineering design errors are not included. However some might be included in the unknown failures used to calculate the overall spacecraft reliability

After the reliabilities were combined in series and system design parameters have been added, a trade-off analysis has been performed. From the study two deorbit strategies emerged as alternatives to the wellknown propulsive de-orbit: drag-augmentation device for nano and microsatellites; $\mathrm{D}$-orbit ${ }^{\mathrm{TM}}$ propulsive device as good alternative for bigger satellites with propulsion. The focus was on the assessment of the impact of spacecraft subsystems on the different deorbit methods, then the strategy selection is left to the satellite's owner who must consider the reliability and safety of the de-orbit method itself.

In addition it is worth noting the need of a constant monitoring and data sharing using a common format for failures attribution at international level. This would improve the knowledge of satellites reliability especially for organizations that are ready to launch many micro and nanosatellites.

\section{$\underline{\text { AKNOWLEDGEMENTS }}$}

The support of Seradata Ltd team in the use of the SpaceTrak $^{\mathrm{TM}}$ database is gratefully acknowledged.

\section{$\underline{\text { APPENDIX }}$}




\begin{tabular}{cccccc}
\hline Mass Class & Total S/C & ATT failures & Mech. failures & TTC failures & POW failures \\
\hline $1-10 \mathrm{~kg}$ Nano & 6 & 0 & 0 & 0 & 1 \\
$10-100 \mathrm{~kg}$ Micro & 53 & 1 & 2 & 0 & 2 \\
$100-500 \mathrm{~kg}$ Mini & 196 & 9 & 7 & 7 & 3 \\
$500-1000 \mathrm{~kg}$ Small & 125 & 17 & 3 & 6 & 6 \\
\hline
\end{tabular}

Table A.1: Spacecraft of the sample equipped with propulsion subsystem and respective number of failures (Class I and II) by typology group.

\begin{tabular}{cccccc}
\hline Mass Class & Total S/C & ATT failures & Mech. failures & TTC failures & POW failures \\
\hline $0.1-1 \mathrm{~kg}$ Pico & 17 & 0 & 1 & 4 & 0 \\
$1-10 \mathrm{~kg}$ Nano & 281 & 3 & 7 & 4 & 10 \\
$10-100 \mathrm{~kg}$ Micro & 100 & 1 & 1 & 4 & 5 \\
$100-500 \mathrm{~kg}$ Mini & 20 & 3 & 1 & 0 & 0 \\
\hline
\end{tabular}

Table A.2: Spacecraft of the sample not equipped with propulsion subsystem and respective number of failures (Class I and II) by typology group.

\section{$\underline{\text { REFERENCES }}$}

[1] IADC, IADC Space Debris Mitigation Guidelines, Steering Group and Working Group 4, 2007.

[2] BSI Standards Publication, ISO 24113:2011 Space systems - Space debris mitigation requirements, 2011.

[3] ESA Director General's Office, ESA/ADMIN/IPOL(2014)2 - Space Debris Mitigation for Agency Projects, 2014.

[4] B. Lazare, The French Space Operations Act: Technical Regulations, Acta Astronaut. 92 (2013) 209-212. doi:http://dx.doi.org/10.1016/j.actaastro.2012.07.031.

[5] L. Innocenti, T. Soares, Clean Space Initiative: Status of Implementation, ESA, 2013.

[6] J.-F. Castet, J.H. Saleh, Beyond reliability, multi-state failure analysis of satellite subsystems: A statistical approach, Reliab. Eng. Syst. Saf. 95 (2010) 311-322. doi:http://dx.doi.org/10.1016/j.ress.2009.11.001.

[7] L. Rossettini, D-Orbit - Solution for our future, in: Tech. Day De-Orbit Strateg., ESTEC, 2015. https://indico.esa.int/indico/event/73/material/0/.

[8] D-Orbit, D-Orbit Decommissioning Device, (2015). http://www.deorbitaldevices.com/site/our-technology/ (accessed August 01, 2015). 
[9] S. Antonetti, L. Rossettini, L. Ferrario, Contributing to Orbital Sustainability with an Independent Decommissioning Device for Satellite and Space Launcher Implementing Space Debris Mitigation Measures, in: 5th CEAS Air Sp. Conf., Delft, 2015.

[10] K.T. Nock, K.M. Aaron, D. McKnight, Removing orbital debris with less risk, J. Spacecr. Rockets. 50 (2013) 365-379. doi:10.2514/1.A32286.

[11] J. Kingston, S. Hobbs, P. Roberts, C. Juanes-Vallejo, F. Robinson, R. Sewell, et al., Use of CYPRES ${ }^{\mathrm{TM}}$ cutters with a Kevlar clamp band for hold-down and release of the Icarus De-Orbit Sail payload on TechDemoSat-1, Acta Astronaut. 100 (2014) 82-93. doi:http://dx.doi.org/10.1016/j.actaastro.2014.03.014.

[12] ESA Education, ESEO mission, (2014). http://www.esa.int/Education/ESEO_mission.

[13] SSTL, EO \& Science Platforms CARBONITE-1, (2015). http://www.sstl.co.uk/Products/EO-SciencePlatforms/CARBONITE-1 (accessed September 01, 2015).

[14] G.D. Krebs, Gunter's Space Page, (2015). http://space.skyrocket.de/ (accessed January 20, 2015).

[15] ESA, Earth Observation Portal, (2015). https://eoportal.org/web/eoportal/satellite-missions (accessed January 20, 2015).

[16] SatFlare, (2015). http://www.satflare.com (accessed January 20, 2015).

[17] Z. Anatoly, RussianSpaceWeb, (2015). http://www.russianspaceweb.com (accessed January 20, 2015).

[18] Seradata SpaceTrak, SPACETRAK User Guide v1.4, (2014). http://www.seradata.com/.

[19] E.L. Kaplan, P. Meier, Nonparametric Estimation from Incomplete Observations Source, J. Am. Stat. Assoc. 53 (1958) 457-481.

[20] G. Tripepi, F. Catalano, L'analisi di sopravvivenza con il metodo di Kaplan-Meier, G. Ital. Di Nefrol. (2004) $540-546$.

[21] J.H. Saleh, J.-F. Castet, Spacecraft Reliability and Multi-State Failures: A Statistical Approach, First Edit, John Wiley and Sons, 2011. doi:10.1002/9781119994077.

[22] J.-F. Castet, J.H. Saleh, Satellite and satellite subsystems reliability: Statistical data analysis and modeling, Reliab. Eng. Syst. Saf. 94 (2009) 1718-1728. doi:http://dx.doi.org/10.1016/j.ress.2009.05.004.

[23] J.P. Bentley, Introduction to reliability and quality engineering, 2nd ed., Harlow : Addison-Wesley, 1999.

[24] ESA Space Debris Mitigation WG, ESSB-HB-U-002, (2015) 1-95. 
Figures in order of appearance on paper

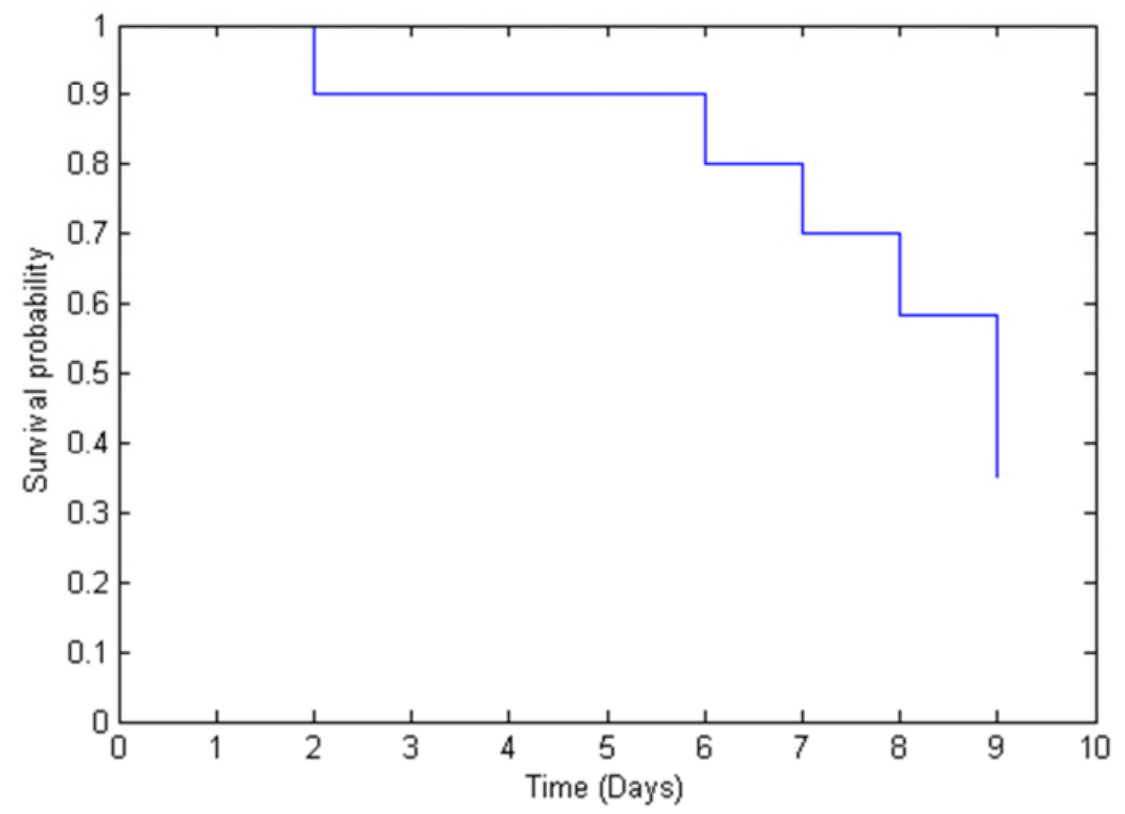

Fig. 1

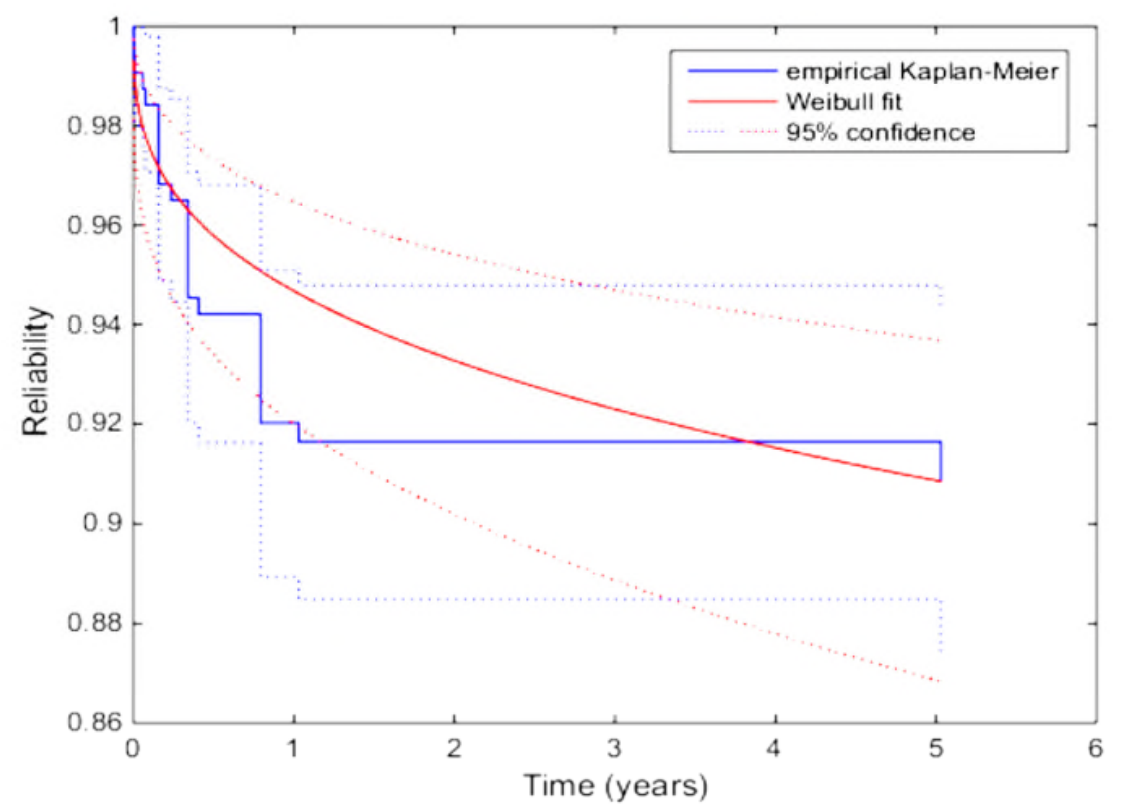

Fig. 2 


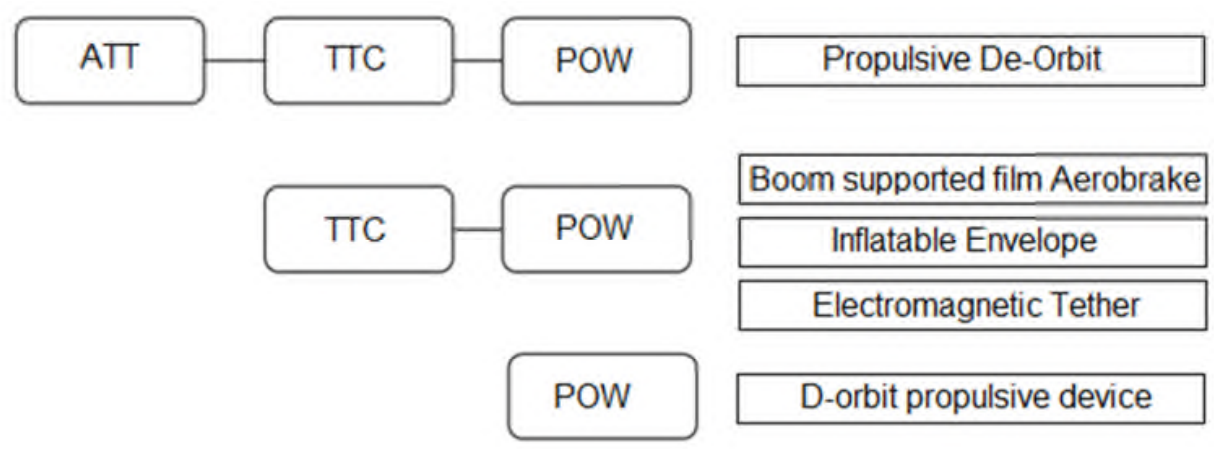

Fig. 3

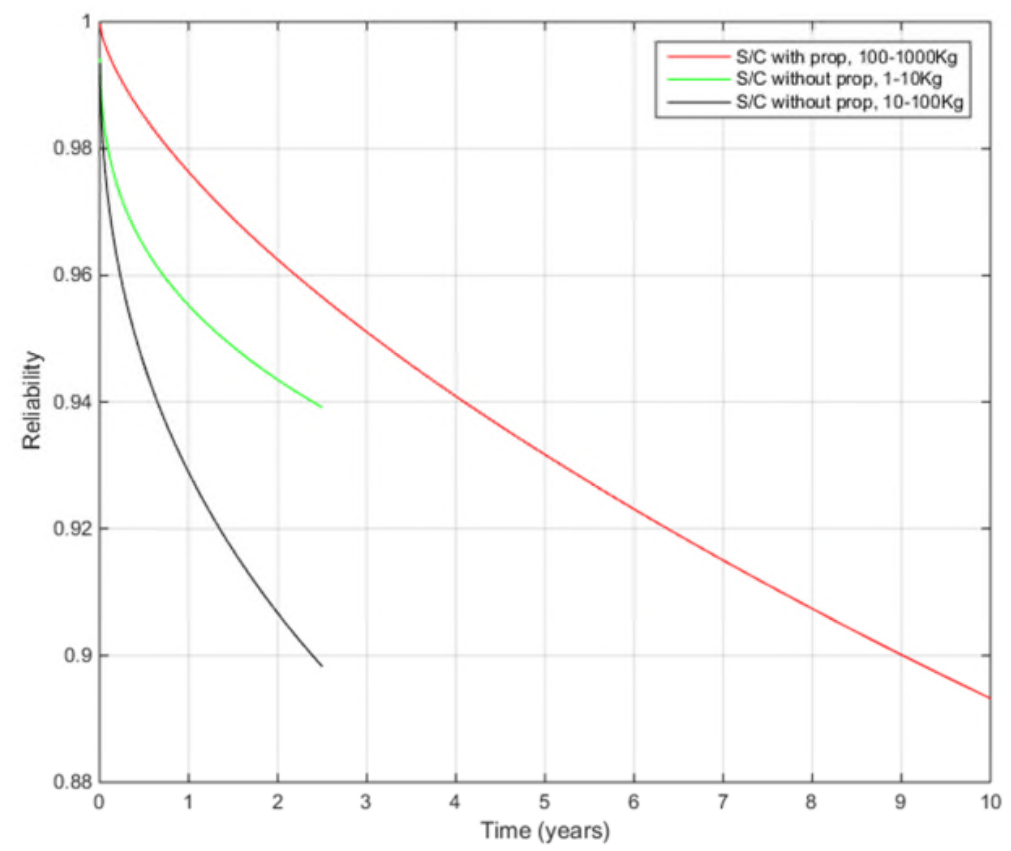

Fig.4 


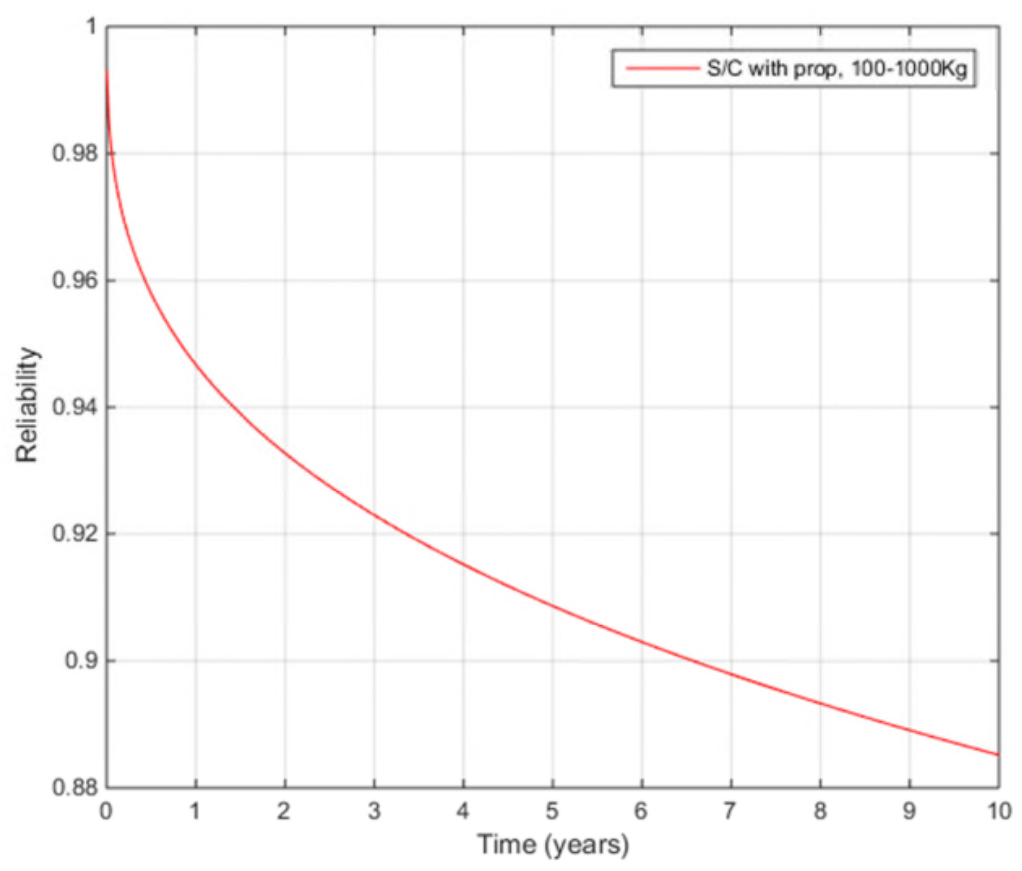

Fig. 5

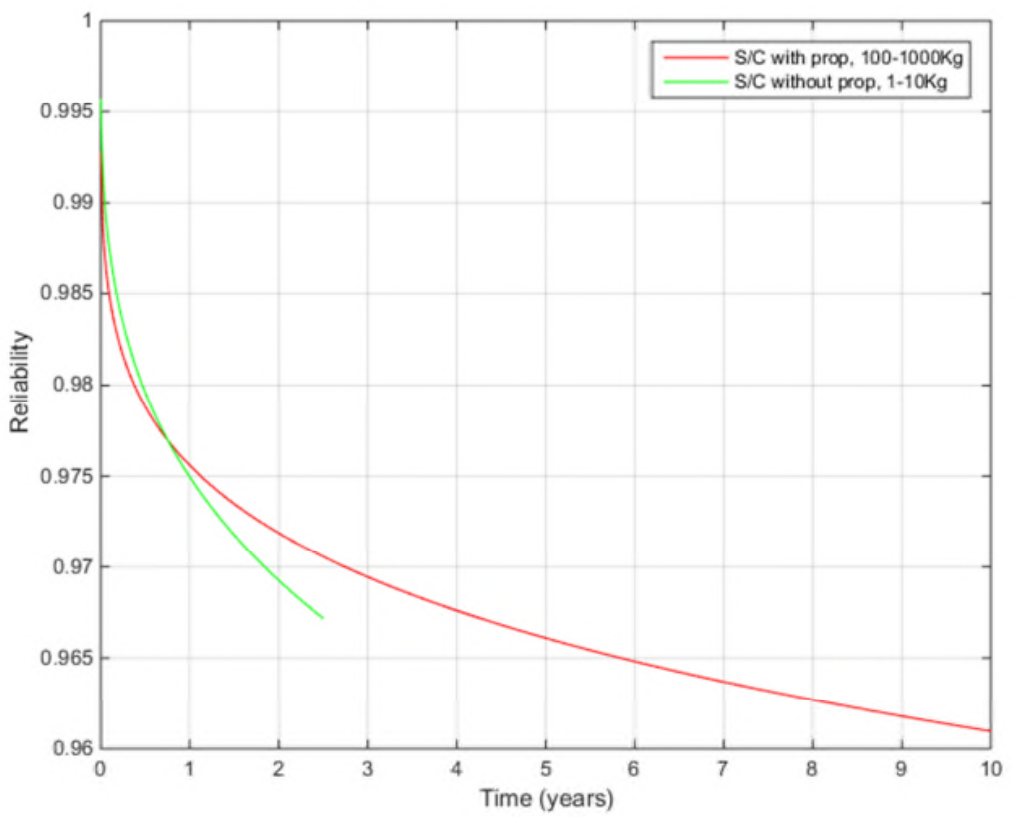

Fig. 6 


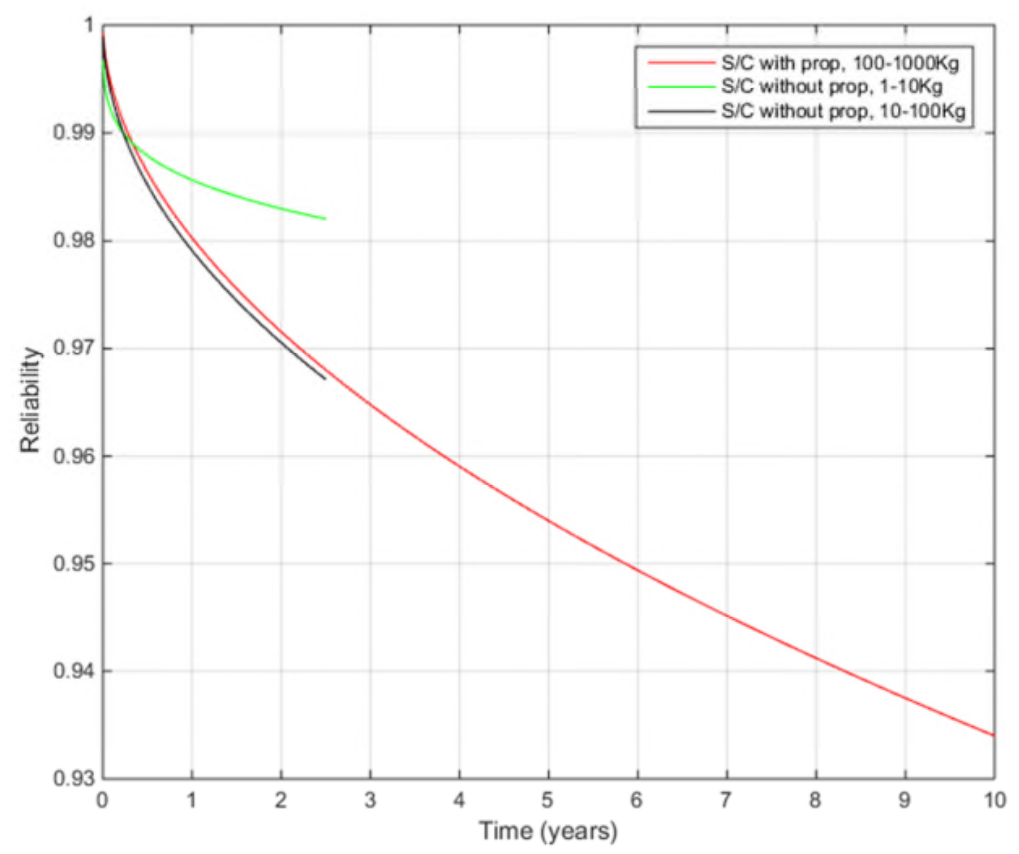

Fig. 7

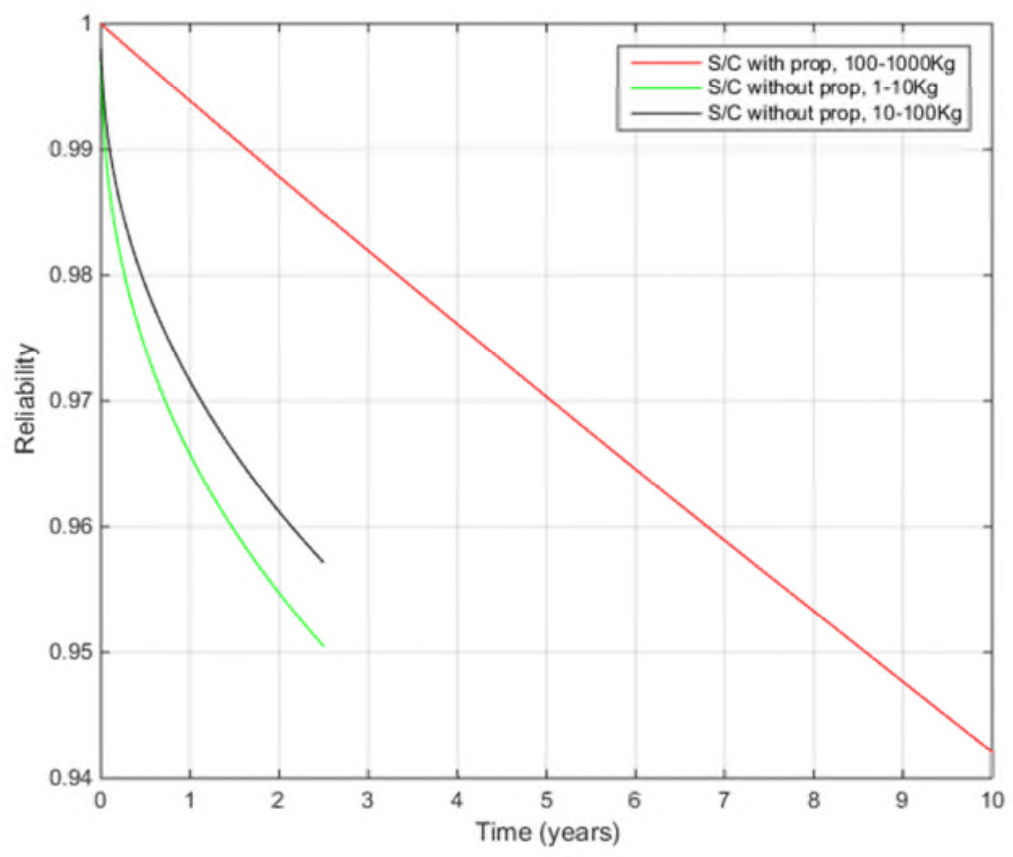

Fig. 8 


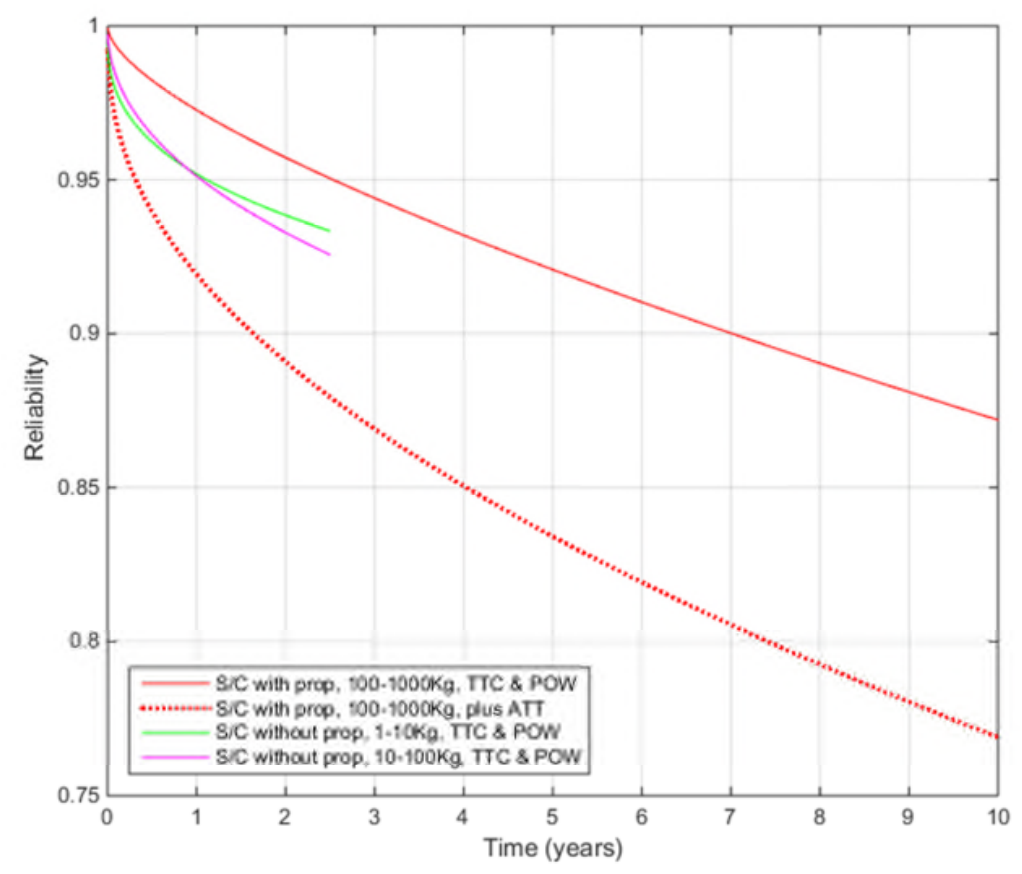

Fig. 9

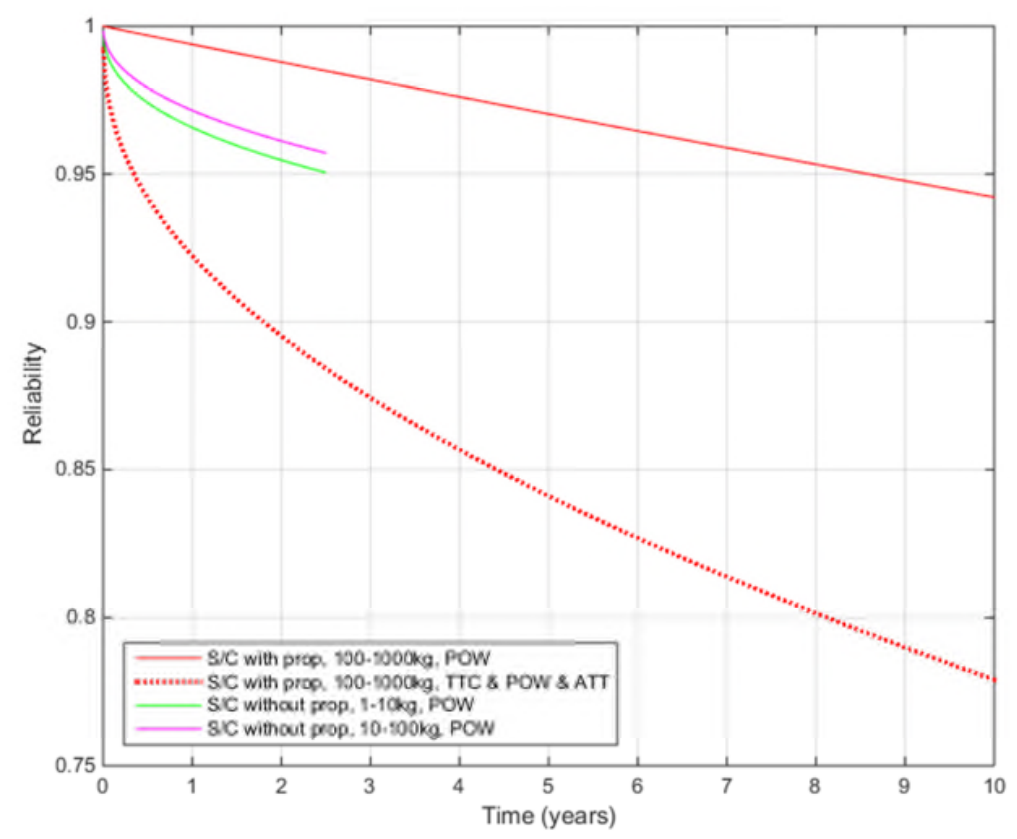

Fig. 10 
2016-07-14

\title{
Failure analysis of satellite subsystems to define suitable de-orbit devices
}

\author{
Palla, Chiara
}

Elsevier

Chiara Palla, Moreno Peroni, Jennifer Kingston, Failure analysis of satellite subsystems to

pÿdefine suitable de-orbit devices, Acta Astronautica, Volume 128, November December 2016, Pages 343-349

http://dx.doi.org/10.1016/j.actaastro.2016.07.021.

Downloaded from Cranfield Library Services E-Repository 